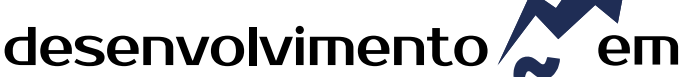 QUESTAOO
}

\section{VIABILIDADE ESTRATÉGICA E ECONÔMICA DE PRÁTICAS SUSTENTÁVEIS NO DEPARTAMENTO DE COMPRAS: Estudo de Caso de Uma IES Privada no Brasil}

\author{
http://dx.doi.org/10.21527/2237-6453.2021.55.11501
}

Recebido em: 17/9/2020

Aceito em: 13/4/2021

\author{
Juan Arturo Castañeda Ayarza, ${ }^{1}$ Denise Helena Lombardo Ferreira, ${ }^{2}$ \\ Cibele Roberta Sugahara, ${ }^{1}$ Antonio Aparecido Jacob ${ }^{1}$
}

\section{RESUMO}

O consumo consciente tornou-se imperativo em sociedades que repensam seu modelo de desenvolvimento. Por exemplo, para uma Instituição de Ensino Superior (IES), que demanda grandes quantidades de materiais, as compras sustentáveis representam uma oportunidade de múltiplos benefícios elencados nas dimensões sociais, ambientais e econômicas. As estratégias sustentáveis de compras, no entanto, podem aumentar a especulação quanto aos efeitos negativos, principalmente aqueles relacionados à parte econômica, fazendo-se necessários estudos que facilitem as tomadas de decisão. Por isso, o objetivo é avaliar a viabilidade estratégica e econômica de práticas sustentáveis no setor de compras de uma IES privada do Brasil. Os resultados mostram a importância de se considerar a sustentabilidade no planejamento estratégico, que deverá estar integrado em todas as áreas da instituição, incluindo o departamento de compras. Pretende-se mostrar, também, que, embora a viabilidade econômica das compras sustentáveis seja fundamental, necessita-se ponderar os impactos econômicos, sociais e ambientais para a instituição.

Palavras-chave: Instituição de Ensino Superior. Compras sustentáveis. Estratégias sustentáveis. Sustentabilidade. Viabilidade econômica.

\section{STRATEGIC AND ECONOMIC VIABILITY OF SUSTAINABLE PURCHASES}

\section{ABSTRACT}

Conscious consumption has become imperative in societies that are rethinking their development model. For example, for a Higher Education Institution (HEI), which demands large quantities of materials, sustainable purchases represent an opportunity for multiple benefits listed in the social, environmental, and economic dimensions. However, sustainable purchasing strategies can increase speculation about the adverse effects, especially those related to the economic part, making it necessary for studies to facilitate decision making. The objective is to assess the strategic and economic feasibility of sustainable practices in the purchasing sector of a private HEI in Brazil. The results show the importance of considering sustainability in strategic planning, which should be integrated in all institution areas, including the purchasing department. Also, although the economic viability of sustainable purchases is fundamental, it is necessary to weigh the economic, social, and environmental impacts for the institution.

Keywords: Higher education institution. Sustainable purchases. Sustainable strategies. Sustainability. Economic viability.

\footnotetext{
${ }^{1}$ Pontifícia Universidade Católica de Campinas (Puccamp).

${ }^{2}$ Autora correspondente. Pontifícia Universidade Católica de Campinas (Puccamp). Rua Professor Dr. Euryclides, de Jesus Zerbini, 1516 - Parque das Universidades. Campinas/SP, Brasil. CEP 13087-571. http://lattes.cnpq.br/5309189687577128. https://orcid. org/0000-0002-3138-2406. lombardo@puc-campinas.edu.br
} 


\section{INTRODUÇÃO}

Em 2015, representantes e líderes dos países que fazem parte das Nações Unidas assinaram a chamada Agenda 2030. Nela foram incluídos 17 Objetivos de Desenvolvimento Sustentável. Embora a Agenda 2030 seja um acordo governamental e intergovernamental, ela também visa a envolver e estimular o setor privado e a sociedade civil para que os objetivos sejam alcançados (JOHNSSON et al., 2020).

Esse cenário desafia as organizações, atingindo todas as atividades da sua cadeia de valor. Por exemplo, na área de compras e suprimentos, tradicionalmente reconhecida a partir do seu potencial de gerar valor financeiro para a organização, pode ser evidenciado o desafio de alinhar suas atividades às pautas da sustentabilidade (JOHNSEN; MIEMCZYK; HOWARD, 2016). Buscando evitar comportamentos oportunistas que derivem no chamado Greenwashing, a organização não governamental WWF publicou, em 2018, um relatório no qual ressalta que as empresas deveriam incluir a pauta da sustentabilidade não somente nas suas próprias operações, mas também nos bens e serviços que são adquiridos ao longo da sua cadeia de valor (VERLES; VELLACOTT, 2018).

A teoria institucional postula que as práticas de gestão ambiental, logo práticas sustentáveis, podem ser motivadas por três tipos de fatores: coercitivo, normativo e mimético (DIMAGGIO; POWELL, 1983). A influência coercitiva vem das forças que estão no poder, como as agências governamentais, e a influência normativa vem das forças exercidas pelos interesses dos stakeholders. As organizações adotam práticas sustentáveis buscando legitimidade organizacional; a influência mimética vem da identificação da necessidade de imitar práticas das organizações concorrentes ou daquelas que alcançaram o sucesso no seu ambiente competitivo (ZHU; GENG, 2013).

No Brasil, a Lei de licitação $n^{\circ} 8.666$ (BRASIL, 1993), interpretada como um fator coercitivo, estipula a necessidade da adoção de critérios sustentáveis nas compras públicas, nas quais, ao adquirir bens e contratar serviços, deve-se priorizar compras locais, cuja produção tenha baixo impacto sobre os recursos naturais e eficiência no uso de água e energia.

A Lei de licitação, no caso das Instituições de Ensino Superior (IES), atinge aquelas que são públicas, mas não as instituições privadas. Cabe ressaltar que, no Brasil, segundo o censo de educação superior de 2018, havia 299 IESs públicas e 2.238 privadas, o que representava quase $88 \%$ do total (INEP, 2019).

As IESs privadas, cujas ações na área de compras sustentáveis advêm de uma medida voluntária, têm tanto, e quiçá em alguns casos até maior, volume de compras de insumos e materiais do que as IESs públicas. Tudo isso, embora não haja dados disponíveis, permite supor o potencial de contribuição aos objetivos do desenvolvimento sustentável por parte do setor de compras das IESs privadas no Brasil.

Nesse sentido, esta pesquisa visa a avaliar, por meio de um estudo de caso, as estratégias e ações pautadas na sustentabilidade, assim como a sua respectiva viabilidade econômica no setor de compras de uma IES privada do Brasil.

A estrutura do artigo apresenta, além desta introdução, o referencial teórico, o método utilizado, a análise dos resultados, as considerações finais e as referências. 


\section{ASPECTOS DAS COMPRAS SUSTENTÁVEIS}

\section{A área de compras e a sustentabilidade}

A gestão de compras sustentáveis é um conceito emergente e amplo, que pode ser entendido como o ato de considerar questões ambientais, sociais, econômicas e éticas para a gestão dos recursos e capacidades externas da organização, para que, quando adquiridos, possam gerar valor não só à organização, mas também à economia e à sociedade (MIEMCZYK; JOHNSEN; MACQUET, 2012).

Na prática, quer dizer que quando uma organização é levada a considerar a sustentabilidade na sua cadeia de suprimento, ela precisará avaliar, de uma forma mais ampla e comple$x a$, o processo de seleção dos seus fornecedores e parceiros (GIUNIPERO; HOOKER; DENSLOW, 2012). Inclusive, como ressaltado por Krause, Vachon e Klassen (2009), uma organização será tão sustentável tanto quanto sustentável forem seus fornecedores.

Hallikas, Lintukangas e Kahkonen (2020) explicam que há um grande e diverso número de ações sustentáveis na gestão da área de compras, e que a introdução dessas práticas dependerá da empresa, dos seus valores, do setor no qual atua, das características das suas compras e da sua base de fornecedores.

Além da seleção de fornecedores e parceiros com base em critérios da sustentabilidade, também é comum que as empresas lancem mão de códigos de conduta, que podem ser entendidos como os padrões utilizados na gestão de relacionamento, no caso pautado na sustentabilidade. Adicionalmente, as empresas utilizam diversos certificados, como a ISO 14001 para a gestão ambiental e a ISO 26000 ou SA8000 para a responsabilidade social. Ainda, pode ser destacado o uso de auditorias externas para garantir a sustentabilidade dos fornecedores (HALLIKAS; LINTUKANGAS; KAHKONEN, 2020).

Organizações que veem a sustentabilidade como uma questão-chave para seu setor de compras, por um lado também deverão avaliar e gerir os riscos nas suas cadeias de suprimentos para evitar danos à sua reputação e à sua economia (HOFMANN et al., 2014). Por outro lado, a empresa deverá avaliar e gerir a sustentabilidade em todas as áreas e níveis da organização e de forma integrada, começando pelo nível estratégico (BARBOSA; CASTAÑEDA-AYARZA; FERREIRA, 2020). Além disso, Baier, Hartmann e Moser (2008) afirmam que, para alcançar os objetivos de desempenho financeiro e não financeiro, será necessário alinhar estrategicamente as prioridades competitivas da organização, como no caso da adoção da sustentabilidade, com as estratégias da área de compras.

Finalmente, pode-se afirmar que as compras sustentáveis contribuem para que organizações com propósitos sustentáveis declarados possam não somente contribuir com a melhora do desempenho sustentável da organização, mas também evitar problemas econômicos e de reputação perante seus stakeholders (BESKE; SEURING, 2014).

\section{Sustentabilidade em Instituições de Ensino Superior}

Para Aleixo, Leal e Azeiteiro (2018), as instituições de ensino superior desempenham papel importante na promoção da sustentabilidade, e as expectativas dos stakeholders para que essas organizações se tornem sustentáveis têm aumentado. Os mesmos autores realizaram uma 
pesquisa exploratória avaliando os principais stakeholders de instituições de Ensino Superior públicas em Portugal, sobre a percepção em relação à sustentabilidade nas Instituições de Ensino Superior. Os resultados mostram, por um lado, que muitos stakeholders não têm clareza quanto ao significado do que seria uma instituição de Ensino Superior sustentável, e, por outro, muitos consideram a questão financeira a principal barreira para o desenvolvimento da sustentabilidade nas instituições.

Embora na literatura não foram encontradas pesquisas que avaliem as compras sustentáveis em instituições de ensino superior privadas, percebeu-se muitos trabalhos que retratam práticas de sustentabilidade em Instituições de Ensino Superior. Alguns exemplos de publicações nacionais e internacionais são relatados na sequência.

Barros (2016), ao referir-se às compras realizadas pelas instituições públicas, destaca que as mesmas são responsáveis pela movimentação de um grande volume de bens e, dessa forma, representam um impacto significativo. No estudo feito para a Universidade Federal de Alfenas, a pesquisadora concluiu que a universidade apresenta práticas sustentáveis por meio de suas compras e contratações, entretanto mostra-se necessário expandir as práticas já realizadas para um número maior de itens, no sentido de manter um padrão de inclusão dos critérios sustentáveis em suas licitações.

Sobre a sustentabilidade nas compras aplicadas à IES, vários outros estudos foram realizados de norte a sul no Brasil, como a pesquisa feita por Bezerra et al. (2011) em duas Instituições Federais de Ensino Superior de Pernambuco, quando investigaram critérios importantes de sustentabilidade para as compras; Cypreste (2013), ao analisar o estágio de operacionalização das compras sustentáveis na Universidade Federal do Espírito Santo; Matias (2014), ao caracterizar as ações socioambientais desenvolvidas na Universidade Federal de Sergipe; Cogo (2015), ao propor a adoção de critérios sustentáveis nas aquisições de bens e contratações de serviços na Gestão Pública na Universidade Tecnológica Federal do Paraná; Viegas e Cabral (2016), na Universidade Federal Rural da Amazônia, ao investigarem a inclusão da temática ambiental na gestão da instituição, incluindo as compras; Freire (2017), na pesquisa realizada com enfoque na sustentabilidade ambiental do Instituto Federal da Paraíba, Campus Aracajú - nesse estudo de caso foram investigados elementos para dar suporte à elaboração do planejamento pedagógico e gestão ambiental da instituição; e Mendonça (2018), ao investigar as compras públicas sustentáveis e suas aplicações nas Instituições Federais de Ensino Superior do Centro-Oeste.

O estudo realizado por Drahein, Lima e Costa (2020) em três Instituições de Ensino Superior da Rede Federal de Educação Profissional, Científica e Tecnológica, discute a importância de definir questões-chave de sustentabilidade para IESs a partir de eixos temáticos. Para a avaliação da sustentabilidade em IESs aplicáveis a operações de serviços, destaca dez temas principais, sendo um deles a temática compras verdes.

Já no âmbito internacional, Clabeaux et al. (2020) avaliou, por meio da análise de ciclo de vida, a atividade que mais contribui com a pegada de carbono no Campus de uma instituição de Ensino Superior no Estados Unidos. Foram avaliadas atividades como: geração de vapor, refrigeração, geração de eletricidade, formas de transporte, tratamento de esgoto e uso de papel. Os resultados mostraram que a geração elétrica é a atividade que mais contribui (41\%) com a emissão de $\mathrm{CO}_{2}$ do Campus. 
Leal Filho et al. (2019) avaliaram o papel dos chamados escritórios verdes ou escritórios sustentáveis como medida que contribui aos esforços pela sustentabilidade dos campi nas Instituições de Ensino Superior. Por meio da uma pesquisa survey em 70 instituições de ensino ao redor do mundo, entendeu-se o processo de instalação e funcionamento dos escritórios verdes e, também, as barreiras e dificuldades ao longo do processo.

Kim, Sunitiyoso e Medal (2019) avaliaram, por meio de um estudo de caso em uma universidade nos Estados Unidos, o processo de tomada de decisão para a elaboração e execução de um projeto de eficiência energética nas construções do campus. Os resultados mostram, além do potencial que a eficiência energética tem na redução das emissões de gases de efeito estufa em uma instituição de ensino, a identificação de cinco importantes categorias que englobam os fatores que influenciam as tomadas de decisão (viabilidade econômica; impacto ambiental; características institucionais; impacto nos usuários dos ambientes do campus; praticidade técnica).

Bekaroo et al. (2019) avaliaram a importância do conhecimento, as atitudes e as ações das pessoas que fazem parte de uma Instituição de Ensino Superior na promoção da sustentabilidade na contribuição com os objetivos sustentáveis da instituição.

Por último, considerou-se importante citar o trabalho de Ramísio et al. (2019), que desenvolveram um método de criação de estratégias sustentáveis em Instituições de Ensino Superior. Os autores ressaltam a importância dos valores e da cultura da organização para o processo de transição em direção à sustentabilidade. Além disso, a proposta considera uma matriz de indicadores que permite controlar o desempenho da sustentabilidade da organização.

Os exemplos relatados evidenciam a adoção de práticas concretas de sustentabilidade em Instituições de Ensino Superior, conseguindo delas se beneficiar e reorganizar suas atividades, combinando sustentabilidade com a geração de oportunidades, como descrito por Veiga (2015).

\section{Obstáculos e benefícios das práticas de compras sustentáveis}

Nas organizações privadas, como uma Instituição de Ensino Superior privado, em que as adesões às pautas da sustentabilidade não são coercitivas, mas normativas e miméticas, a avaliação dos benefícios e também dos obstáculos das práticas de compras sustentáveis acaba sendo importante, mas, ao mesmo tempo, suscetível às percepções do mercado e do agente comprador.

O Quadro 1 destaca os principais obstáculos do ponto de vista do comprador e do mercado em relação à prática de compras sustentáveis.

Entre os obstáculos identificados, pode-se ressaltar a "percepção de maiores custos, por não contemplar o benefício econômico futuro" na visão do comprador e, na visão do mercado, a "falta de apoio gerencial por desconhecerem a relevância da prática de compras sustentáveis". Estes podem ser fatores decisivos no descarte e não aprovação de estratégias sustentáveis no setor de compras.

No Quadro 2 destacam-se os benefícios das práticas de compras sustentáveis na visão do comprador e do mercado. Entre os benefícios identificados, ressalta-se a "promoção do desenvolvimento de comunidades locais e sua infraestrutura física, gerando retornos sociais (empre- 
VIABILIDADE ESTRATÉGICA E ECONÔMICA DE PRÁTICAS SUSTENTÁVEIS NO DEPARTAMENTO DE COMPRAS: ESTUDO DE CASO DE UMA IES PRIVADA NO BRASIL

Juan Arturo Castañeda Ayarza - Denise Helena Lombardo Ferreira - Cibele Roberta Sugahara - Antonio Aparecido Jacob

gabilidade)" na visão do comprador e, na visão do mercado, não foi possível identificar um fator preponderante que pudesse ser ressaltado.

Quadro 1 - Obstáculos para as práticas de compras sustentáveis

\begin{tabular}{l|l}
\hline \multicolumn{2}{c}{ Visão do comprador } \\
\hline $\begin{array}{l}\text { Percepção de maiores custos, por não contemplar o benefício } \\
\text { econômico futuro. }\end{array}$ & $\begin{array}{l}\text { Preuss (2007); Walker e Brammer } \\
\text { (2009); Zhu, Geng e Sarkis (2013) }\end{array}$ \\
\hline $\begin{array}{l}\text { Em razão da baixa oferta de fornecedores que oferecem produtos } \\
\text { e serviços sustentáveis, os preços elevam-se pela falta de } \\
\text { competitividade. }\end{array}$ & Seixas (2018) \\
\hline $\begin{array}{l}\text { Ofertas insuficientes de produtos. } \\
\text { Falta de conhecimento sobre meio ambiente por parte dos } \\
\text { fornecedores. }\end{array}$ & Walker e Jones (2012) \\
\hline Poucos fornecedores de bens ou serviços sustentáveis. & Korkmaz (2010); Testa et al. (2012) \\
\hline
\end{tabular}

Visão do mercado

Falta de informação e de experiência do comprador/consumidor para fazer a comparação das características de um produto específico.

Dificuldade, por parte dos compradores, para definir quais produtos e serviços são considerados sustentáveis, uma vez que os compradores não são especialistas ambientais.

Falta de apoio gerencial por desconhecerem a relevância da prática de compras sustentáveis.

lads (2008)

Carência de experiência e de capacidade técnica dos profissionais de compras para a introdução dos critérios de sustentabilidade nas aquisições.

Falta de informações a respeito do mercado de produtos sustentáveis, por exemplo, materiais utilizados, processo de fabricação, reciclagem e ciclo de vida.

Falta de motivação dos envolvidos no processo de compras e de cultura organizacional orientada às práticas de aquisições sustentáveis.

Bouwer et al. (2011)

Bouwer et al. (2011); Luthra et al.

(2011); Walker e Jones (2012); Zhu, Geng e Sarkis (2013)

Bouwer et al. (2011)

Brammer e Walker (2011)

Al Zaabi, Al Dhaheri e Diabat (2013)

Fonte: Elaboração própria. 
VIABILIDADE ESTRATÉGICA E ECONÔMICA DE PRÁTICAS SUSTENTÁVEIS NO DEPARTAMENTO DE COMPRAS: ESTUDO DE CASO DE UMA IES PRIVADA NO BRASIL

Juan Arturo Castañeda Ayarza - Denise Helena Lombardo Ferreira - Cibele Roberta Sugahara - Antonio Aparecido Jacob

Quadro 2 - Benefícios das práticas de compras sustentáveis - visão do comprador

\begin{tabular}{|c|c|}
\hline \multicolumn{2}{|l|}{ Visão do comprador } \\
\hline $\begin{array}{l}\text { Ganho reputacional por atuar ativamente na proteção socioambiental: "imagem } \\
\text { positiva". }\end{array}$ & $\begin{array}{l}\text { Biderman et al.(2008); } \\
\text { ICLEI (2015) }\end{array}$ \\
\hline $\begin{array}{l}\text { Contribuição para superar os desafios ambientais: degradação do solo, perda de } \\
\text { biodiversidade, acesso à água, dentre outros. }\end{array}$ & Iclei (2015) \\
\hline $\begin{array}{l}\text { Estímulo aos mercados locais e globais para inovar e produzir produtos e serviços } \\
\text { mais sustentáveis para compradores e consumidores organizacionais públicos e } \\
\text { privados. }\end{array}$ & Seixas (2018) \\
\hline $\begin{array}{l}\text { Promoção do desenvolvimento de comunidades locais e sua infraestrutura física, } \\
\text { gerando retornos sociais (empregabilidade). }\end{array}$ & $\begin{array}{l}\text { Biderman et al. } \\
\text { (2008); ICLEI (2015); } \\
\text { Seixas (2018) }\end{array}$ \\
\hline Aumento da conscientização sobre temas socioambientais pela comunidade local. & Roos (2012) \\
\hline $\begin{array}{l}\text { Contribuição com o fluxo de caixa futuro (produtos com eficiência energética } \\
\text { favorecem futura economia de recursos financeiros). }\end{array}$ & Preuss (2007) \\
\hline \multicolumn{2}{|l|}{ Visão do mercado } \\
\hline $\begin{array}{l}\text { Ao se fazer compras locais tem-se como consequência a redução dos custos } \\
\text { relacionados com o transporte, o combustível utilizado, a manutenção dos veículos } \\
\text { e os congestionamentos rodoviários. }\end{array}$ & Roos (2012) \\
\hline $\begin{array}{l}\text { Mercados condutores para soluções inovadoras, pois as compras sustentáveis } \\
\text { podem induzir os mercados a adotarem tecnologias mais limpas, estimulando a } \\
\text { competitividade e, finalmente, a redução dos preços em razão das economias de } \\
\text { escala. }\end{array}$ & Biderman et al.(2008) \\
\hline $\begin{array}{l}\text { Redução do impacto ambiental (efeitos sobre a saúde e bem-estar) e redução do } \\
\text { uso de recursos (reduzir, reciclar, reutilizar) decorrente da aquisição sustentável. }\end{array}$ & Biderman et al. (2008) \\
\hline $\begin{array}{l}\text { Produtos com eficiência energética economizam recursos financeiros e emitem } \\
\text { menos } \mathrm{CO}_{2} \text {. }\end{array}$ & Iclei (2015) \\
\hline
\end{tabular}

Fonte: Elaboração própria.

\section{MÉTODO}

Esta pesquisa caracteriza-se como qualitativa descritiva com estudo de caso. O estudo qualitativo, segundo Richardson (1999, p. 80), permite investigar situações complexas e particulares e pode ser aplicado quando se almeja "contribuir no processo de mudança de determinado grupo".

A pesquisa adota a estratégia do estudo de caso que, de acordo com Yin (2010, p. 39-40), refere-se a uma pesquisa empírica em profundidade com enfoque em evento contemporâneo. A investigação do estudo de caso conta com várias fontes de evidências e "beneficia-se do desenvolvimento anterior das proposições teóricas para orientar a coleta e a análise de dados".

Quanto à delimitação do objeto, o estudo de caso desta pesquisa é uma Instituição de Ensino Superior privada localizada no Estado de São Paulo, Brasil. Para a seleção da unidade de análise desta pesquisa utilizou-se, como critério, o recomendado por Yin (2010) quanto às 
proposições específicas do estudo de caso. Trata-se de uma IES cujo aspecto peculiar das ações pautadas na sustentabilidade na área de compras, carecia de uma análise da viabilidade das práticas sustentáveis alinhadas com um plano estratégico. Seguindo os procedimentos relatados por Yin (2010, p. 41), o estudo de caso preocupa-se em "descrever uma intervenção e o contexto da vida real no qual ela ocorreu".

A escolha da IES do estudo de caso deste trabalho justifica-se considerando que ela possui identidade católica e comunitária, e, no aspecto compras sustentáveis, procura nortear a execução de suas atividades pelos seguintes valores: compromisso social, responsabilidade com o meio ambiente e desenvolvimento com sustentabilidade econômico-financeira.

Em relação ao procedimento para a coleta de dados, a presente pesquisa procedeu com a coleta de dados primários e secundários. Os dados primários foram coletados a partir da observação dos procedimentos adotados junto aos representantes do departamento de Engenharia da IES estudada, e também a partir dos conhecimentos de um dos pesquisadores deste estudo que atua na área de compras da IES.

Os dados secundários foram obtidos a partir da busca de evidências relatadas em estudos anteriores publicados em livros, artigos, dissertações e teses e fontes de dados dos sites das empresas, seus portfólios e catálogos de acesso público, que serviram de base para realizar o levantamento de informações e valores dos serviços de locação dos veículos elétricos e das lâmpadas fluorescentes e de LED. Para complementar as informações foram consultados documentos internos, como orçamentos e documentos dos fornecedores de posse do departamento de compras da IES estudada.

Como estratégia de análise dos dados foram realizadas duas etapas:

a) Análise estratégica (qualitativa e descritiva, baseada na teoria) das ações encontradas no setor de compras sustentáveis da IES escolhida, que busca responder às seguintes perguntas: Quais estratégias são utilizadas ou pretendem ser empregadas pelo setor de compras? As estratégias selecionadas fizeram parte de um processo de planejamento? As estratégias selecionadas estão alinhadas e integradas à estratégia principal ou geral da organização?

b) Análise da viabilidade econômica das estratégias da IES escolhida e, também, análise do impacto ambiental das estratégias. Espera-se responder: As estratégias escolhidas pela IES são economicamente viáveis? Há ou espera-se algum tipo de impacto das estratégias de compras sustentáveis nas questões ambientais e/ou energéticas na IES?

\section{RESULTADOS}

\section{As estratégias da IES em estudo}

Um dos autores deste estudo exerce atividades no setor de compras da IES escolhida para o estudo de caso, o que permitiu ter acesso a dados específicos que fazem parte das atividades do setor, como requisição de materiais; dados sobre os fornecedores; orçamentos apresentados pelos fornecedores; mapa comparativo de preços; e os pedidos de compra.

Lembrando-se que a IES em estudo é privada e não tem motivadores coercitivos para assumir práticas sustentáveis nas suas atividades, inicialmente identificou-se que a instituição 
tem declarado formalmente, na sua postura estratégica, valores alinhados com as pautas da sustentabilidade, como compromisso social, responsabilidade com o meio ambiente e desenvolvimento com sustentabilidade econômico-financeira.

Apesar de serem evidenciadas práticas que condizem com os valores anunciados em relação à sustentabilidade, não foi explicitado um plano estratégico que tenha a sustentabilidade como foco em todas as áreas da instituição, incluindo a sustentabilidade do campus e a sustentabilidade nas atividades na área de compras. Nesse sentido, também não foi identificado um plano estratégico, pautado na sustentabilidade, próprio da área de compras.

Em relação às práticas de compras sustentáveis, no entanto, existem duas propostas que ainda não estavam na qualidade de estratégias pretendidas no momento da pesquisa. A primeira estratégia tem a intensão de substituir todas as lâmpadas fluorescentes do campus por lâmpadas de LED. A segunda estratégia visa a fazer a locação de veículos elétricos para as diversas necessidades de transporte dentro do campus.

\section{A viabilidade das estratégias pretendidas pelo setor de compras}

\section{Substituição das fluorescentes por LED}

No campus, majoritariamente, é feito o uso da lâmpada fluorescente tubular $1200 \mathrm{~mm}$ T8 32W. A intenção é trocar pela lâmpada de LED (Light Emitting Diode) modelo CorePro Tube $1200 \mathrm{~mm} 18$ W 840 T8C W G. A Tabela 1 apresenta características desses dois tipos de lâmpadas.

Tabela 1 - Lâmpada fluorescente versus lâmpada de LED

\begin{tabular}{lcc}
\hline & Fluorescente & LED \\
\hline Vida útil em horas & 15000 & 30000 \\
Quantidade de horas acesas por dia (média) & 14 & 14 \\
Quantidade de dias úteis por mês & 22 & 22 \\
Quantidade de horas acesas por mês (média) & 308 & 308 \\
Custo unitário (R\$) (setembro 2019) & 8,00 & 18,88 \\
Potência (Wh) & 33,6 & 18 \\
Custo substituição (R\$) & $\mathrm{N} / \mathrm{D}(1)$ & $\mathrm{N} / \mathrm{D}$ \\
Custo descarte unitário (R\$) (setembro 2019) & 0,95 & $\mathrm{~N} / \mathrm{D}$ \\
Quantidade em análise & 1 & 1 \\
Frequência troca (anos) & 4 & 8 \\
Horas ligadas (h/ano) & 3696 & 3696 \\
Consumo energia (KWh/ano) & 124 & 67 \\
Custo KWh (R\$̦/KWh) (setembro 2019) & 0,64 & 0,64 \\
\hline
\end{tabular}

(1) Não Disponível

Fonte: Elaboração própria.

Com base nas características da lâmpada fluorescente apresentada na Tabela 1, foram realizados os cálculos para a obtenção do gasto anual da lâmpada, considerando-se as variáveis aquisição, descarte e custo da energia elétrica (Tabela 2). 
VIABILIDADE ESTRATÉGICA E ECONÔMICA DE PRÁTICAS SUSTENTÁVEIS NO DEPARTAMENTO DE COMPRAS: ESTUDO DE CASO DE UMA IES PRIVADA NO BRASIL

Juan Arturo Castañeda Ayarza - Denise Helena Lombardo Ferreira - Cibele Roberta Sugahara - Antonio Aparecido Jacob

Tabela 2 - Lâmpada fluorescente - aquisição, descarte e custo anual da energia elétrica

\begin{tabular}{ccccc}
\hline \multicolumn{4}{c}{ Situação Corrente - Fluorescente (em Reais) } & \\
\hline Ano & Aquisição & $\begin{array}{c}\text { Descarte } \\
\text { Fluorescente }\end{array}$ & Custo Energia & Total Anual \\
\hline 0 & 0,00 & 0,00 & 0,00 & 0,00 \\
1 & 0,00 & 0,00 & $-79,48$ & $-79,48$ \\
2 & 0,00 & 0,00 & $-79,48$ & $-79,48$ \\
3 & 0,00 & 0,00 & $-79,48$ & $-79,48$ \\
4 & $-8,00$ & $-0,95$ & $-79,48$ & $-88,43$ \\
5 & 0,00 & 0,00 & $-79,48$ & $-79,48$ \\
6 & 0,00 & 0,00 & $-79,48$ & $-79,48$ \\
7 & 0,00 & 0,00 & $-79,48$ & $-79,48$ \\
8 & $-8,00$ & $-0,95$ & $-79,48$ & $-88,43$ \\
9 & 0,00 & 0,00 & $-79,48$ & $-79,48$ \\
10 & 0,00 & 0,00 & $-79,48$ & $-79,48$ \\
\hline Total & & & & $-812,69$ \\
\hline
\end{tabular}

Fonte: Elaboração própria.

Conforme os dados apresentados, ao final de dez anos a lâmpada fluorescente tem um gasto de aproximadamente $R \$ 812,69$, considerando a aquisição e descarte da lâmpada e o custo da energia elétrica. Deve-se ressaltar que o descarte da lâmpada acontece a cada quatro anos.

Na Tabela 3 expõe-se os cálculos sobre os gastos com a aquisição da lâmpada LED, descarte da fluorescente e o custo da energia elétrica que seria consumida. O resultado mostra que, ao substituir as lâmpadas fluorescentes pelas lâmpadas de LED, o gasto com a energia elétrica tem uma queda de $\mathrm{R} \$ 79,48$ (Tabela 2 ) para $\mathrm{R} \$ 42,58$ (Tabela 3 ) por ano. Por outro lado, conforme dados obtidos no catálogo da Philips (c2018-2019), a lâmpada de LED tem vida útil de aproximadamente 30 mil horas, ou seja, duas vezes maior que a vida útil da lâmpada fluorescente. Ao final de dez anos a lâmpada de LED ocasionaria um gasto aproximado de $R \$ 464,49$, considerando-se a compra das lâmpadas de LED e o descarte das lâmpadas fluorescentes. A Tabela 4 destaca a economia gerada ao substituir a lâmpada fluorescente pela lâmpada de LED.

Tabela 3 - Lâmpada de LED - aquisição e gasto com energia e descarte da fluorescente

\begin{tabular}{ccccc}
\hline \multicolumn{4}{c}{ Situação Proposta - LED (em Reais) } \\
\hline Ano & Aquisição & $\begin{array}{c}\text { Descarte } \\
\text { Fluorescente }\end{array}$ & Custo Energia & Total Anual \\
\hline 0 & $-18,88$ & $-0,95$ & 0,00 & $-19,83$ \\
1 & 0,00 & 0,00 & $-42,58$ & $-42,58$ \\
2 & 0,00 & 0,00 & $-42,58$ & $-42,58$ \\
3 & 0,00 & 0,00 & $-42,58$ & $-42,58$ \\
4 & 0,00 & 0,00 & $-42,58$ & $-42,58$ \\
5 & 0,00 & 0,00 & $-42,58$ & $-42,58$ \\
\hline
\end{tabular}




\begin{tabular}{ccccc}
\hline 6 & 0,00 & 0,00 & $-42,58$ & $-42,58$ \\
7 & 0,00 & 0,00 & $-42,58$ & $-42,58$ \\
8 & $-18,88$ & 0,00 & $-42,58$ & $-61,46$ \\
9 & 0,00 & 0,00 & $-42,58$ & $-42,58$ \\
10 & 0,00 & 0,00 & $-42,58$ & $-42,58$ \\
\hline Total & & $-464,49$ \\
\hline
\end{tabular}

Fonte: Elaboração própria.

Tabela 4 - VP - Substituição da lâmpada fluorescente pela lâmpada de LED

\begin{tabular}{cccccc}
\hline \multicolumn{5}{c}{ Delta (Situação Proposta - Situação Corrente) (em Reais) } \\
\hline Ano & Aquisição & $\begin{array}{c}\text { Substituição e } \\
\text { Descarte }\end{array}$ & Custo Energia & Total Anual & $\begin{array}{c}\text { Valor Presente } \\
\text { (VP) }\end{array}$ \\
\hline 0 & $-18,88$ & $-0,95$ & 0,00 & $-19,83$ & $-19,83$ \\
1 & 0,00 & 0,00 & 36,90 & 36,90 & 33,55 \\
2 & 0,00 & 0,00 & 36,90 & 36,90 & 30,50 \\
3 & 8,00 & 0,95 & 36,90 & 45,85 & 34,45 \\
4 & 0,00 & 0,00 & 36,90 & 36,90 & 25,20 \\
5 & 0,00 & 0,00 & 36,90 & 36,90 & 22,91 \\
6 & 8,00 & 0,95 & 36,90 & 45,85 & 25,88 \\
7 & 0,00 & 0,00 & 36,90 & 36,90 & 18,94 \\
8 & $-18,88$ & 0,00 & 36,90 & 18,02 & 8,41 \\
9 & 8,00 & 0,95 & 36,90 & 45,85 & 19,45 \\
10 & 0,00 & 0,00 & 36,90 & 36,90 & 14,23 \\
\hline \multicolumn{7}{c}{} & & & Total (VP)==> & 213,67 \\
\cline { 4 - 5 }
\end{tabular}

Fonte: Elaboração própria.

De acordo com a Tabela 4, ao trazer para o Valor Presente (VP), utilizando a taxa real de desconto anual de $10 \%$, para o período de dez anos, a substituição da lâmpada fluorescente pela lâmpada de LED resultaria em uma economia aproximada, por lâmpada, de $R \$ 213,67$ ao Valor Presente. Ao decidir pela substituição das lâmpadas em salas de aula, laboratórios e bibliotecas, seriam descartadas aproximadamente 15 mil lâmpadas e a economia, ao longo de dez anos, ao Valor Presente, poderia ser de $\mathrm{R} \$ 3.205 .050,00$, ou seja, $\mathrm{R} \$ 320.505,00$ por ano.

Ao considerar o preço unitário da lâmpada de LED, $R \$ 18,88$ (Tabela 1), e a possibilidade de substituição de 15.000 lâmpadas, a despesa inicial seria de $R \$ 283.200,00$ ( $R \$ 18,88 * 15.000$ $=R \$ 283.200,00)$.

Conforme a Tabela 2, em dez anos uma lâmpada fluorescente gastará aproximadamente $R \$ 812,69$, ou $R \$ 81,27$ por ano e $R \$ 6,77$ por mês $(R \$ 812,69 / 10=R \$ 81,27 / 12=R \$ 6,77)$.

De acordo com a Tabela 3, a lâmpada de LED possui uma eficiência energética e vida útil maior ao ser comparada com a lâmpada fluorescente. Em dez anos a lâmpada de LED teria um gasto aproximado de $R \$ 464,49$, ou $R \$ 46,45$ por ano e $R \$ 3,87$ por mês ( $R \$ 464,49 / 10=R \$$ $46,45 / 12=R \$ 3,87)$. 
Vale destacar que, ao substituir a lâmpada fluorescente por lâmpada de LED, é possível obter uma economia mensal aproximada de $R \$ 2,90$ por lâmpada ( $R \$ 6,77-R \$ 3,87=R \$ 2,90)$. Isso gera a economia de $\mathrm{R} \$ 43.525,00$ por mês para 15.000 lâmpadas $(\mathrm{R} \$ 2,90 * 15.000=\mathrm{R} \$$ 43.525,00).

Ao considerar a despesa inicial para a aquisição das lâmpadas de LED de $R \$ 283.200,00$ e a economia mensal de $\mathrm{R} \$ 43.525,00$, pode-se afirmar que essa despesa poderá ser amortizada em seis meses e meio ( $R \$ 283.200,00 / R \$ 43.525,00=6,5)$.

A lâmpada de LED tem um custo $136 \%$ superior ao da lâmpada fluorescente, porém, após dez anos, seu gasto representa aproximadamente $57,15 \%$ do gasto da lâmpada fluorescente e, além disso, a lâmpada de LED tem uma vida útil quase duas vezes maior que o da fluorescente.

No que respeita ao impacto ambiental, iniciando pelo consumo de energia, a lâmpada de LED consumirá 16 Watts a menos por lâmpada fluorescente, ou $4.805 \mathrm{Wh} / \mathrm{mês} /$ lâmpada. Isso quer dizer que em dez anos as 15 mil lâmpadas de LED terão economizado o consumo equivalente a 8.648.640 KWh. Por outro lado, as lâmpadas de LED não contêm, entre sua matéria-prima, o metal pesado mercúrio, presente nas lâmpadas fluorescentes.

O aspecto desfavorável para que a IES estudada considere substituir as lâmpadas de LED pelas fluorescentes, é que não há uma regulamentação para o correto descarte. Cabe lembrar que a lâmpada de LED é composta de metais, componentes eletrônicos, plástico, vidro, terras raras e elementos químicos, dentre os quais o metal pesado chumbo, utilizado para fixar o diodo de LED na placa de iluminação (LIM et al., 2011).

\section{Locação de veículos elétricos}

A estratégica de locação de veículos elétricos é a que necessita de maior aprofundamento de estudos quanto à sua adoção e alcance.

Entende-se que a ideia principal é substituir os atuais veículos que a IES possui, que utilizam motor movido à combustão interna, por veículos elétricos, que, no caso específico, seriam alugados. A Tabela 5 apresenta informações de cotação de preços para a locação dos veículos elétricos. Vale destacar que nesse momento a IES não tem uma grande quantidade de fornecedores. Os fornecedores A (Rio de Janeiro-RJ) e B (Vinhedo-SP) são de médio para grande porte, e o fornecedor C (São Paulo-SP) de médio para pequeno porte.

Ao considerar o fornecedor com o menor preço, isto é, fornecedor A, a locação de um veículo elétrico modelo Carryall Cargo Box, com capacidade para dois passageiros e $300 \mathrm{~kg}$ de carga, apresenta o custo mensal de $R \$ 2.200,00$, totalizando um gasto anual de $R \$ 26.400,00$ (Tabela 5).

Tabela 5 - Cotação de preços de locação do veículo elétrico (em reais)

\begin{tabular}{ccccc}
\hline & Quant. & \multicolumn{3}{c}{ Fornecedores } \\
\cline { 3 - 5 } & & A & B & C \\
\hline Veículo elétrico de modelo Carryall Cargo Box & 1 & & & \\
Total mês para o comprador & & $2.200,00$ & $2.228,00$ & $2.600,00$ \\
Total ano para o comprador & & $26.400,00$ & $26.736,00$ & $31.200,00$ \\
\hline
\end{tabular}

Fonte: Elaboração própria. 
Com a finalidade de comparar o gasto entre o veículo elétrico e o veículo com motor movido à combustão interna, será utilizado o modelo VW Up com motor à combustão interna, por apresentar condições similares de atendimento à demanda interna da IES quando comparado com o modelo elétrico Carryall Cargo Box.

Conforme o departamento de transportes da IES em estudo, o modelo de veículo VW Up tem um custo anual aproximado de $\mathrm{R} \$ 10.700,00$. Esse valor leva em conta os custos com depreciação, combustível, manutenção, seguro, licenciamento e DPVAT.

Tendo em vista que o custo anual do veículo com motor movido à combustão interna é de $\mathrm{R} \$ 10.700,00$ e do veículo elétrico de $\mathrm{R} \$ 26.400,00$, pode-se afirmar que a opção de locação de veículos elétricos não é economicamente viável.

No que respeita à questão ambiental, foi abordado o impacto no consumo energético. Para isso, considerou-se que um veículo percorre aproximadamente $19.200 \mathrm{~km} / \mathrm{ano}$ no campus da IES. Isso quer dizer que, em um ano, um veículo elétrico consumirá o equivalente a 7.796 $\mathrm{KWh} / \mathrm{ano}$, enquanto um veículo à combustão interna, utilizando gasolina, consumirá o equivalente a $16.457 \mathrm{KWh} /$ ano e um veículo utilizando etanol consumirá o equivalente a $17.170 \mathrm{KWh} /$ ano. Ou seja, um veículo elétrico poderá economizar, em um ano, entre $53 \%$ e $55 \%$ da energia consumida por um veículo à combustão interna. Esses dados evidenciam a contribuição deste tipo de veículo elétrico em termos ambientais.

Além disso, o veículo elétrico apresenta outras vantagens, como: fácil dirigibilidade; o condutor não precisa de habilitação; não emite qualquer tipo de gases de efeito estufa e nem de material particulado durante sua utilização; e reduz a poluição sonora (INTERNATIONAL ENERGY AGENCY, 2013; FONTAÍNHAS, 2013).

Finalmente, a estratégia de locação dos veículos elétricos, além de ocasionar maior custo para a IES, tem como desvantagem a baixa potência do motor e a exigência de que seu uso será apenas dentro do campus, pois o veículo elétrico avaliado não está regulamentado pelo órgão do Departamento de Trânsito (Detran) quanto às questões de emplacamento.

\section{DISCUSSÃO DOS RESULTADOS}

A partir da abordagem estratégica, as implicações práticas deste estudo para a IES analisada sugerem a necessidade de deliberar um plano estratégico que permita alinhar os valores declarados pela instituição e todas as estratégias utilizadas em todos os níveis e departamentos da estrutura organizacional. Essa necessidade é sustentada pelos trabalhos de Baier, Hartmann e Moser (2008), Hitt, Ireland e Hoskisson (2018) e Barbosa, Castañeda-Ayarza e Ferreira (2020). Inclusive, seria viável avaliar experiências de outras IESs com a sustentabilidade e os processos e métodos utilizados na elaboração, efetivação e controle de estratégias sustentáveis, como encontrado no trabalho de Ramísio et al. (2019).

Dentro do departamento de compras, a proposta e adoção de estratégias sustentáveis não deverá ser elaborada de forma emergente, mas deliberada, avaliando técnica e amplamente o alinhamento das propostas do setor com as expectativas e principais estratégias da IES. Além disso, considera-se fundamental que as estratégias sustentáveis na área de compras levem em conta os riscos financeiros e não financeiros, como o alinhamento estratégico entre os valores da IES e os valores das empresas parceiras e fornecedoras que serão escolhidas. Essa 
observação está em consonância com os achados de Giunipero, Hooker e Denslow (2012), Krause, Vachon e Klassen (2009), Hallikas, Lintukangas e Kahkonen (2020) e Hofmann et al. (2014).

Embora no departamento de compras a questão financeira seja, tradicionalmente, o tipo de controle aplicado às ações realizadas (PREUSS, 2007; WALKER; BRAMMER, 2009; ZHU; GENG; SARKIS, 2013), quando se fala em compras sustentáveis (sendo a sustentabilidade uma opção voluntária, influenciada pela normatividade e a mimética) além da viabilidade econômica será necessário avaliar o peso do impacto que poderão ter as estratégias de compras sustentáveis na imagem e na qualidade de relacionamento da IES com seus stakeholders (internos e externos). Ou seja, como mostrado por Kim, Sunitiyoso e Medal (2019), a tomada de decisão sobre a sustentabilidade em todas as atividades de uma IES deverá levar em consideração a viabilidade econômica, o impacto ambiental, as características institucionais, o impacto nos usuários dos ambientes do campus e a praticidade técnica da ação sustentável.

Ainda, é possível ressaltar que a decisão pela inserção das práticas de compras sustentáveis no processo de compras da IES pode contribuir com a exigência do Objetivo 12 da ONU, que trata de assegurar padrões de produção e consumo sustentáveis (ONU, 2015).

Por outro lado, os resultados da análise econômica das duas estratégias pretendidas pelo departamento de compras da IES estudada mostram que as estratégias deveriam ser elaboradas em razão das necessidades internas da organização, além da disponibilidade de produtos e serviços sustentáveis e da acessibilidade a fornecedores e empresas parceiras. Lembra-se que, como apresentado por Korkmaz (2010) e Testa et al. (2012), além da questão econômica, os principais obstáculos para as práticas sustentáveis no departamento de compras são a disponibilidade e o acesso a produtos e serviços sustentáveis.

Embora a estratégia pretendida pela IES, de substituir as lâmpadas fluorescentes do campus pelas lâmpadas de LED, seja claramente viável em termos econômicos, nem toda estratégia sustentável terá uma vantagem expressiva nesse quesito. O departamento de compras deverá propor e ponderar opções estratégicas que equilibrem os benefícios econômicos, ambientais e sociais (BIDERMAN et al., 2008; ICLEI, 2015; SEIXAS, 2018; PREUSS, 2007). Isso quer dizer que, no caso da estratégia que pretende substituir veículos de combustão interna por veículos elétricos, a possibilidade não deverá ser abandonada, mas reformulada, avaliando opções diferentes à locação, que, talvez, tenham um impacto econômico menos negativo ou então mais equilibrado entre as questões econômicas, sociais e ambientais.

Cabe reforçar, como mostrado por Bekaroo et al. (2019), que o perfil econômico de qualquer opção de compra sustentável poderá ser melhorado na medida em que as estratégias de aquisição de produtos e serviços sustentáveis estejam alinhadas ou, desde outra perspectiva, que haja estratégias sustentáveis sendo praticadas em outras áreas da instituição e por todos os integrantes dela. Por exemplo, pode-se mencionar o uso e o consumo consciente da energia elétrica, da água, das máquinas, dos insumos e dos produtos que possui a IES.

\section{CONSIDERAÇÕES FINAIS}

Embora a IES analisada não tenha um plano estratégico geral e setorial claro pautado na sustentabilidade, este estudo conseguiu contribuir com o entendimento da importância estratégica do planejamento pautado na sustentabilidade, do alinhamento entre estratégias de toda a 
organização e da necessidade de que o departamento de compras pondere os impactos econômicos das estratégias sustentáveis junto com os impactos sociais e ambientais.

Ficou evidente, também, o quão importante é, para a sustentabilidade de uma organização, a sustentabilidade dos seus fornecedores e parceiros, os stakeholders, e, nesse desafio, a importância que tem o departamento de compras.

Desde o ponto de vista acadêmico, observou-se a necessidade de mais estudos que avaliem e proponham alternativas gerais e específicas para inserir a sustentabilidade em Instituições de Educação Superior privadas, que, no Brasil, não têm um motivador coercitivo, mas voluntário. Assim, assumir voluntariamente o desfio de tornar sustentável uma IES dependerá da capacidade de avaliar e entender as pressões dos interesses de cada stakeholder, assim como da capacidade de identificar as necessidades e oportunidades para a sustentabilidade de todas as atividades e recursos da organização.

Finalmente, desde o ponto de vista prático, sugere-se que a IES avaliada considere a possibilidade de elaborar planos estratégicos departamentais alinhados a um plano estratégico institucional pautados na sustentabilidade, tendo em vista que a sustentabilidade está implícita no contexto de um ideal de desenvolvimento sustentável.

\section{REFERÊNCIAS}

ALEIXO, A. M.; LEAL, S.; AZEITEIRO, U. M. Conceptualization of sustainable higher education institutions, roles, barriers, and challenges for sustainability: An exploratory study in Portugal. Journal of Cleaner Production, v. 20, p. 1.664-1.673, 2018.

AL ZAABI, S.; AL DHAHERI, N.; DIABAT, A. Analysis of interaction between the barriers for the implementation of sustainable supply chain management. The International Journal of Advanced Manufacturing Technology, v. 68, n. 1/4, p. 895-905, 2013.

BAIER, C.; HARTMANN, E.; MOSER, R. Strategic alignment and purchasing efficacy: an exploratory analysis of their impact on financial performance. Journal of Supply Chain Management, v. 44, n. 4, p. 36-52, 2008. BARBOSA, M.; CASTAÑEDA-AYARZA, J. A.; FERREIRA, D. H. L. Sustainable Strategic Management (GES): Sustainability in small business. Journal of Cleaner Production, v. 258, p. 120880, 2020.

BARROS, I. O. Compras públicas sustentáveis: um estudo na Universidade Federal de Alfenas-MG. 2016. 74 f. Dissertação (Mestrado em Administração Pública) - Universidade Federal de Alfenas. Disponível em: https://200.131.224.39:8443/bitstream/tede/933/5/Disserta\%C3\%A7\%C3\%A30\%20de\%20lsabela\%20 Oliveira\%20Barros.pdf. Acesso em: 18 nov. 2020.

BEKAROO, G.; BOKHOREE, C. H.; RAMSAMY, P.; MOEDEEN, W. Investigating personal carbon emissions of employees of higher education institutions: Insights from Mauritius. Journal of Cleaner Production, v. 209, p. 581-594, 2019.

BESKE, P.; SEURING, S. Putting sustainability into supply chain management. Supply Chain Management: Int. J., v. 19, n. 3, p. 322-331, 2014.

BEZERRA, M. S. C. et al. Critérios de sustentabilidade nas decisões de compras realizadas no setor público: um estudo multicaso em duas Ifes do Estado de Pernambuco. CONGRESSO ANPCONT, 2011. São Paulo. Anais [...]. São Paulo, 2011.

BIDERMAN, R.; MACEDO, L. S. V. de; MONZONI, M.; MAZON, R (org.). Guia de compras públicas sustentáveis: uso do poder de compra do governo para a promoção do desenvolvimento sustentável. 2. ed. Rio de Janeiro: FGV, 2008.

BOUWER, M.; JONK, M.; SZUPPINGER, P.; LUSSER, H.; BERMAN, T.; BERSANI, R.; NAPPA, V.; VIGANÒ, C.; NISSIEN, A.; PARIKKA, K. Green Public Procurement in Europe 2006: Conclusions and recommendations. Haarlem: Virage Milieu \& Management, 2011. 
VIABILIDADE ESTRATÉGICA E ECONÔMICA DE PRÁTICAS SUSTENTÁVEIS NO DEPARTAMENTO DE COMPRAS: ESTUDO DE CASO DE UMA IES PRIVADA NO BRASIL

Juan Arturo Castañeda Ayarza - Denise Helena Lombardo Ferreira - Cibele Roberta Sugahara - Antonio Aparecido Jacob

BRAMMER, S.; WALKER, H. Sustainable procurement in the public sector: An international comparative study. International Journal of Operations and Production Management, v. 31, n. 4, 452-476, 2011.

BRASIL. Lei no 8.666, de 21 de junho de 1993. Regulamenta o art. 37, inciso XXI, da Constituição Federal, institui normas para licitações e contratos da Administração Pública e dá outras providências. Brasília: Presidência da República. Disponível em: http://www.planalto.gov.br/ccivil_03/Leis//8666cons.htm\#targetText=Art.,Par\%C3\%A1grafo\%20\%C3\%BAnico. Acesso em: 31 maio 2019.

CLABEAUX, R.; CARBAJALES-DALE, M.; LADNER, D.; WALKER, T. Assessing the carbon footprint of a university campus using a life cycle assessment approach. Journal of Cleaner Production, v. 273, 2020. Doi: https://doi.org/10.1016/j.jclepro.2020.122600

COGO, G. A. R. Critérios de sustentabilidade nas aquisições de bens e contratações de serviços da Gestão Pública Federal. 2015. 111 f. Dissertação (Mestrado em Engenharia de Produção) Universidade Tecnológica Federal do Paraná, 2015.

CYPRESTE, A. S. T. Licitações sustentáveis instrumento legal de promoção da sustentabilidade: um estudo da aquisição de bens na Universidade Federal do Espírito Santo Ufes. 2013. 107 f. Dissertação (Mestrado Profissional em Gestão Pública) - Universidade Federal do Espírito Santo, Vitória, 2103.

DIMAGGIO, P. J.; POWELL, W. W. The iron cage revisited: institutional isomorphism and collective rationality in organizational fields. American Sociological Review, v. 48, n. 2, p. 147-160, apr., 1983.

DRAHEIN, A. D.; LIMA, E. P.; COSTA, S. E. G. Ferramentas para avaliação da sustentabilidade nas operações de serviço em instituições de Ensino Superior. Revista de Gestão Ambiental e Sustentabilidade - GeAS, v. 9, n. 1, p. 1-25, 2020.

FONTAÍNHAS, J. J. C. Avaliação de viabilidade económica da aquisição de um veículo elétrico em Portugal. 2013. 87 f. Dissertação (Mestrado em Engenharia Industrial) - Universidade do Minho, Braga, 2013.

FREIRE, J. J. A. Avanços e desafios da sustentabilidade ambiental no Instituto Federal de Sergipe: um estudo de caso no Campus Aracajú. 2017. 113 f. Dissertação (Mestrado em Desenvolvimento e Meio Ambiente) - Universidade Federal de Sergipe, João Pessoa, 2017.

GIUNIPERO, L. C.; HOOKER, R.E.; DENSLOW, D. Purchasing and supply management sustainability: drivers and barriers. J. Purch. Supply Manag, v. 18, n. 4, p. 258-269, 2012.

HALLIKAS, J.; LINTUKANGAS, K.; KAHKONEN, A. K. The effects of sustainability practices on the performance of risk management and purchasing. Journal of Cleaner Production, v. 263, 2020. Doi:10.1016/j. jclepro.2020.121579.

HITT, M. A.; IRELAND, R. D.; HOSKISSON, R. E. Administração estratégica: competitividade e globalização: conceitos. 12. ed. São Paulo: Cengage, 2018.

HOFMANN, H.; BUSSE, C.; BODE, C.; HENKE, M. Sustainability-related supply chain risks: conceptualization and management. Bus. Strat. Environ, v. 23, n. 3, p. 160-172, 2014.

IADS. Instituto Argentino para el Desarrollo Sustentable. Proyecto Piloto Compras Públicas Sustentables en el Mercosur. Las Compras Públicas en el Mercosur: Propuesta para la incorporación de criterios de sustentabilidad. Buenos Aires: lads, 2008. Disponível em: http://www.iadsargentina.org/pdf/Documento_base.pdf. Acesso em: 6 maio 2019.

ICLEI. Governos Locais para a Sustentabilidade. Manual Procurat: um guia para implementação de compras públicas sustentáveis. 3. ed. São Paulo: Iclei Brasil, 2015. Disponível em: http://www.procurement-forum.eu/resource/download/653/manual-procura-br-final.pdf. Acesso em: 2 maio 2019.

INEP. Instituto Nacional de Estudos e Pesquisas Educacionais Anísio Teixeira. Censo da Educação Superior 2018: notas estatísticas. Brasília: Inep, 2019.

INTERNATIONAL ENERGY AGENCY. Global EV Outlook: Understanding the electric vehicle landscape to 2020. [S.I.]: IEA, 2013. Disponível em: https://www.iea.org/publications/freepublications/publication/ GlobalEVOutlook_2013.pdf. Acesso em: 5 set. 2019.

JOHNSEN, T. E.; MIEMCZYK, J.; HOWARD, M. A systematic literature review of sustainable purchasing and supply research: Theoretical perspectives and opportunities for IMP-based research. Industrial Marketing Management, 2016. 
VIABILIDADE ESTRATÉGICA E ECONÔMICA DE PRÁTICAS SUSTENTÁVEIS NO DEPARTAMENTO DE COMPRAS: ESTUDO DE CASO DE UMA IES PRIVADA NO BRASIL

Juan Arturo Castañeda Ayarza - Denise Helena Lombardo Ferreira - Cibele Roberta Sugahara - Antonio Aparecido Jacob

JOHNSSON, F.; KARLSSON, I.; ROOTZÉN, J.; AHLBACK, A.; GUSTAVSSON, M. The framing of a sustainable development goals assessment in decarbonizing the construction industry - Avoiding "Greenwashing". Renewable and Sustainable Energy Reviews, v. 131, 2020. Doi: https://doi.org/10.1016/j.rser.2020.110029 KIM, A. A.; SUNITIYOSO, Y.; MEDAL, L. A. Understanding facility management decision making for energy efficiency efforts for buildings at a higher education institution. Energy and Buldings. v. 15, p. 197-215, 2019.

KORKMAZ, A. Benefits and obstacles of environmental, social and sustainable procurement. Seul: IPPC4, 2010. Disponível em: http://www.ippa.org/IPPC4/Proceedings/07GreenProcurement/Paper7-2.pdf. Acesso em: 6 maio 2019.

KRAUSE, D. R.; VACHON, S.; KLASSEN, R. D. Special topic forum on sustainable supply chain management: Introduction and reflections on the role of purchasing management. Journal of Supply Chain Management, v. 45, n. 4, p. 18-25, 2009.

LEAL FILHO, W.; WILL, M.; SALVIA, A. L.; ADOMBENT, M.; GRAHL, A.; SPIRA, F. The role of green and Sustainability Offices in fostering sustainability efforts at higher education institutions. Journal of Cleaner Production, v. 20, p. 1.394-1.401, 2019.

LIM, S. R.; KANG, D.; OGUNSEITAN, O. A.; SCHOENUNG, J. M. Potential environmental impacts of Light-Emitting Diodes (LEDs): Metallic resources, toxicity, and Hazardous Waste Classification. Environmental Science \& Technology, v. 45, n. 1, p. 320-327, 2011.

LUTHRA, S.; KUMAR, V.; KUMAR, S.; HALEEN, A. Barriers to implement green supply chain management in automobile industry using interpretive structural modeling technique-An Indian perspective. Journal of Industrial Engineering and Management, v. 4, n. 2, p. 231-257, 2011.

MATIAS, S. L. A. Responsabilidade socioambiental na Universidade Federal de Sergipe: princípios e práticas para a promoção da sustentabilidade. 2014. 136 f. Dissertação (Mestrado em Desenvolvimento e Meio Ambiente) - Universidade Federal de Sergipe, 2014.

MENDONÇA, C. T. P. C. A sustentabilidade no processo de compras em Instituições Federais de Ensino Superior do Centro-Oeste. 2018. 144 f. Dissertação (Mestrado em Administração Pública Pofiap) - Universidade Federal de Goiás, Goiânia, 2018.

MIEMCZYK, J.; JOHNSEN, T. E.; MACQUET, M. Sustainable purchasing and supply management: A structured literature review of definitions and measures at dyad, chain and network levels. Supply Chain Management: An International Journal, v. 17, n. 5, p. 478-496, 2012.

ONU. Organização das Nações Unidas. Objetivo 12: consumo e produção responsáveis. Assegurar padrões de produção e de consumo sustentáveis. [S.I.]: ONU, 2015. Disponível em: https://nacoesunidas.org/ pos2015/ods12/. Acesso em: 10 out. 2019.

PREUSS, L. Buying into our future: Sustainability initiatives in local government procurement. Business Strategy and the Environment, v. 16, n. 5, p. 54-365, 2007.

RAMÍSIO, P. J.; PINTO, L. M. C.; GOUVEIA, N., COSTA, H.; AREZES, D. Sustainability Strategy in Higher Education Institutions: Lessons learned from a nine-year case study. Journal of Cleaner Production, v. 222, p. 300-309, 2019.

RICHARDSON, R. J. Pesquisa social: métodos e técnicas. São Paulo: Atlas, 1999.

ROOS, R. Sustainable Public Procurement: Briefing Note. [S.I.]: UNDP, 2012. Disponível em: http://www. unpcdc.org/media/390120/spp_brief_en_2012-02-06.pdf. Acesso em: 6 maio 2019.

SEIXAS, E. S. Governança nas compras públicas sustentáveis: um modelo para os Institutos Federais de Educação, Ciências e Tecnologia baseado na análise de redes sociais. 2018. 183 f. Tese (Doutorado Multi-institucional e Multidisciplinar em Difusão do Conhecimento) - Universidade Federal da Bahia, Salvador, 2018.

TESTA, F.; IRALDO, F.; FREY, M.; DADDI, T. What factors influence the uptake of GPP (green public procurement) pratices? Ecological Economics, v. 82, p. 88-96, 2012.

VEIGA, J. E. da. Para entender o desenvolvimento sustentável. São Paulo: Editora 34, 2015.

VERLES, M.; VELLACOTT, T. Business and the Sustainable Development Goals: Best practices to seize opportunity and maximise credibility. Gold Standard and WWF Report, 2018.

VIEGAS, S. F.; CABRAL, E. R. Adesão de uma universidade pública à agenda ambiental na administração pública-A3P. CODS-Colóquio Organizações, Desenvolvimento e Sustentabilidade, v. 5, n. 1, p. 241-263, 2016. 
WALKER, H.; BRAMMER, S. Sustainable procurement in the United Kingdom public sector. Supply Chain Management, v. 14, n. 2, p. 128-137, 2009.

WALKER, H.; JONES, N. Sustainable supply chain management across the UK private sector. Supply Chain Management, v. 17, n. 1, p. 15-28, 2012.

ZHU, Q.; GENG, Y. Drivers and barriers of extended supply chain practices for energy saving and emission reduction among Chinese manufacturers. Journal of Cleaner Production, v. 40, p. 6-12, 2013.

ZHU, Q.; GENG, U.; SARKIS, J. Motivating green public procurement in China: An individual level perspective. Journal of Environmental Management, v. 126, p. 85-95, 2013.

YIN, R. K. Estudo de caso: planejamento e métodos. 4. ed. Porto Alegre: Bookman, 2010. 